\title{
Transcriptome Sequencing of Trichoderma Koningiopsis Strain Tk1 to Identify and Analyze Differential Gene Expression Patterns
}

Mei Luo

Innovative Institute for Plant Health, Zhongkai University of Agriculture and Engineering, Guangzhou, China

Zhangyong Dong ( $\square$ dongzhangyong@hotmail.com )

Innovative Institute for Plant Health, Zhongkai University of Agriculture and Engineering, Guangzhou, China https://orcid.org/0000-0001-7524-0226

\section{Yongxin Shu}

Zhongkai University of Agriculture and Engineering

\section{Mobing Chen}

Zhongkai University of Agriculture and Engineering

\section{Research article}

Keywords: Trichoderma koningiopsis, Biocontrol, Carbohydrate-active enzyme, Differential gene expression analysis

Posted Date: August 20th, 2020

DOI: https://doi.org/10.21203/rs.3.rs-55968/v1

License: (c) (i) This work is licensed under a Creative Commons Attribution 4.0 International License. Read Full License 


\section{Abstract}

Background: Trichoderma koningiopsis strain Tk1 shows good biocontrol potential. However, its biocontrol function may differ under different conditions. The objective of this study is to elucidate the biological and transcriptome differences of $T$. koningiopsis Tk1 under different media.

Results: In this study, the mycelium weight and sporulation of T. koningiopsis Tk1 was found to differ in various media. Further, the Tk1 strain inhibited the growth of the pathogen Fusarium oxysporum in the three media tested. Fries3, PD, and PS were collected for RNA sequencing of Tk1 mycelia to identify the genes that are differentially expressed genes (DEGs) between Tk1 grown on different media. De novo transcriptome assembly resulted in identification of 14,208 unigenes. The differential gene expression pattern was more similar between the Fries3 and PS samples, whereas PD samples showed a different expression pattern. The DEGs were enriched in some metabolic and biosynthetic pathways. Additional analysis of the DEGs identified a set of carbohydrate-active enzymes that are upregulated or downregulated under different conditions.

Conclusions: These results indicate that the Tk1 strain cultured in Fires3 and PS mediums can produce specific metabolic and carbohydrate-active enzymes to enhance their antimicrobial effect, providing a foundation for the subsequent mining of specific genes.

\section{Background}

Trichoderma is a genus of fungi that is widely distributed in nature and used effectively in biocontrol to treat a range of pathogens worldwide [1,2]. More than 250 species of Trichoderma have been identified [3] and strains of $T$. koningiopsis [4, 5] show great potential in the biocontrol of pathogens. The mechanisms of biocontrol by Trichoderma sp. include competition for space and nutrients [6], mycoparasitism [7], antibiosis [8], promotion of plant growth [9], enhanced nutrient solubility and subsequent acquisitions of minerals [8], and induction of systemic resistance [10,11].

Carbohydrate-active enzymes (CAZymes), particularly cell wall-degrading enzymes, affect the mycoparasitic properties of Trichoderma sp. For instance, the enzymes $\beta-1,3$-glucanase and protease were produced when $T$. harzianum mycelia were transferred from a medium containing glucose as the sole carbon source to medium containing cell walls of Botrytis cinerea [12]. Silva et al. [13] reported that 10 different chitinase producing Trichoderma strains varied in their enzyme activity, with higher chitinase activity resulting in a stronger inhibitory effect towards the pathogen.

During the Second World War, American canvas tents in the Solomon Islands were disintegrated by the cellulase enzyme produced by $T$. reesei. T. lignorum was first introduced because of its ability to attack and control plant pathogenic fungi [14]. Trichoderma sp. including T. koningiopsis have been widely explored and used as biocontrol agents in many important cash crops. T. koningiopsis Th003 is an efficient biocontrol agent of different plant pathogens and can induce systemic resistance against $F$. oxysporum in tomato [15]. T. koningiopsis YIM PH30002 was shown to inhibit the growth of some tested 
phytopathogenic fungi including Phoma herbarum, Fusarium flocciferum, Scytalidium lignicola, and Epicoccum nigrum [1]. Substances such as koninginins $\mathrm{B}, \mathrm{E}, \mathrm{J}, \mathrm{N}-\mathrm{Q}$, and 7-O-methylkoninginin $\mathrm{D}$ have been isolated from the YIM PH30002 strain [16]. T. koningiopsis has shown great potential for the biocontrol of phytopathogenic fungi; different species or different strains of the same species show different control effects. Therefore, it is important to identify effective biocontrol strains. Based on the available scientific literature, T. koningiopsis Tk1 shows potential as a biocontrol strain.

The production of antibiotic compounds depends on several factors, such as fungal growth phase and nutritional, biological, and environmental conditions; these fungi can be induced or activated by other organisms when they invade the soil and compete for available nutrients, in addition to various other mechanisms [17]. The volatile and nonvolatile secondary metabolites of Trichoderma play a significant and effective role in plant pathogen suppression and plant growth promotion [18]. Different types of antibiotic metabolites have been isolated from Trichoderma. For instance, the volatile pyranone 6-pentyl$2 \mathrm{H}$-pyran-2-one has been isolated from some species of Trichoderma [19-22] and exerts a strong inhibitory effect against Rhizoctonia solani, Fusarium oxysporum, and B. cinerea [8]. This pyranone produced by $T$. asperellum ismT 5 induces a plant resistance response and inhibits leaf diseases caused by $B$. cinerea and Alternaria sp.

Some CAZymes and antibiotic metabolites secreted by Trichoderma sp. can act synergistically [23]. The number and functions of CAZymes and antifungal substances produced vary between species, strains, and culture conditions. Horta et al. [23] identified a set of CAZymes that are upregulated under different conditions based on their T. harzianum IOC-3844 expression. Bech et al. [24] demonstrated that the production of cell wall-degrading enzymes by $T$. asperellum varied based on the cellulosic material available. Most published research on Trichoderma has been conducted using a single liquid-state or solid-state fermentation, whereas little information is available on multiple culture conditions.

Recently, a strain of T. koningiopsis Tk1 was isolated in Guangdong, China from the root of the medicinal plant Chrysanthemum morifolium "Huangju” [25]. In this study, T. koningiopsis Tk1 cultured in three different media was tested for its biological characteristics and antimicrobial effects. Mycelia collected from three growth media were subjected to RNA sequencing and analyzed for their differential gene expression (DEG) patterns.

\section{Results}

\section{Mycelium growth and sporulation of Tk1 strain in different media}

The mycelium weight of $T$. koningiopsis Tk1 differed between different media. The mycelium weights of the Tk1 strain grown for $7 \mathrm{~d}$ in Fries3, PD, and PS media were $1.73,1.29$, and $0.94 \mathrm{~g}$, respectively (Figure 1A). After $7 \mathrm{~d}$, the spore pair values in the Fries3, PD, and PS media were 8.6, 8.4, and 8.3, respectively (Figure 1B).

Filtrate antifungal bioassay 
The axenic fermentation extracts of $T$. koningiopsis Tk1 from different media were obtained. Both the extract and media were added to the PDA medium. The results showed that the media did not significantly impact the mycelial growth of $F$. oxysporum. Moreover, the axenic fermentation extract in the three media differed. A total of $3 \mathrm{~mL}$ axenic fermentation extract was added to $7 \mathrm{~mL}$ PDA for each test. Both axenic fermentation extracts from Fries3 and PS media showed better inhibition of pathogenic $F$. oxysporum. After $3 \mathrm{~d}$, the pathogen colony diameters in plates with extract added from Fries3, PS, and PD media were $23.5 \mathrm{~mm}$ (inhibition rate of $22.95 \%$ ), $20.25 \mathrm{~mm}$ (inhibition rate of $33.61 \%$ ), and $30.00 \mathrm{~mm}$ (inhibition rate of 1.64\%), respectively; the pathogen colony diameter in PDA was $30.50 \mathrm{~mm}$. After $5 \mathrm{~d}$, the colony diameter in plates with extract added from Fries3, PS, and PD media were $45.00 \mathrm{~mm}$ (inhibition rate of $33.58 \%$ ), $44.50 \mathrm{~mm}$ (inhibition rate of $34.32 \%$ ), and $58.75 \mathrm{~mm}$ (inhibition rate of $13.28 \%$ ), respectively; the pathogen colony diameter in CK was $67.75 \mathrm{~mm}$ (Figure 2).

\section{Sequencing assembly}

Averages of $58,150,778,61,910,742$, and $48,690,148$ raw reads were obtained from sequencing the Fries3, $\mathrm{PD}$, and PS cDNA libraries of $T$. koningiopsis Tk1, respectively. After trimming adaptor sequences and eliminating low-quality reads, $56,134,944,59,723,474$, and $46,770,465$ clean reads were obtained from Fries, PD, and PS samples, respectively (Supplementary Table S1). After combined assembly, the datasets resulted in 14,208 unigenes with a mean length of 2124 base pairs (bp), N50 of 4001 bp, and N90 of 803 bp (Supplementary Table S2).

\section{Functional annotation of the assembled unigenes}

Annotation was conducted using the BLASTx and BLASTn programs with an E-value cut-off of $10^{-5}$. A total of 12,201 (85.87\%) unigenes was annotated by at least one of the databases: $10,796(75.98 \%)$ unigenes were annotated by the NCBI-NR database, 3025 (21.29\%) unigenes by the KO database, 11,158 (78.53\%) unigenes by the NCBI-Nt database, 8614 (60.62\%) by Swiss-Prot, 8748 (61.57\%) unigenes by the PFAM database, 8748 (61.57\%) unigenes by the GO database, and 3817 (26.86\%) by the KOG database (Figure 3A). BLASTx homology search in the NCBI-NR database showed that T. koningiopsis transcriptomes had the best blast match to Trichoderma sequences, primarily to T. gamsii (43\%) and T. atroviride (19.7\%) (Figure 3B). All unigenes in the T. koningiopsis transcriptomes were subjected to GO analysis for the three categories molecular function, biological process (BP), and cellular component (CC) (Figure 3C). In the molecular function (MF) GO category, the most abundant transcripts were linked to binding and catalytic activity. In the BP category, the most enriched term was cellular process, with 5532 unigenes. The metabolic process and single-organism process terms were also abundant. In the CC category, cell and cell parts were the most enriched terms.

\section{Analysis of DEGs}

The square of the Pearson correlation coefficient $\left(R^{2}\right)$ was used to measure the correlation between samples. The $\mathrm{R}^{2}$ values for Fries $3, \mathrm{PD}$, and PS samples were $0.959,0.964$, and 0.917 , respectively (Supplementary Figure S1). 
DEGs were normalized using the reads per kilobase of transcript per million mapped reads method. Compared with Fries3, PD showed 2618 DEGs, of which 1416 were upregulated and 1202 were downregulated. Compared with PS, PD showed 2293 DEGs, of which 1120 were upregulated and 1173 were downregulated. Compared with Fries, PS showed 876 DEGs, of which 382 were upregulated and 494 were downregulated (Figure 4A). Comparison of the DEGs from the Fries3 vs PD group and from the PS vs PD group revealed 1520 common DEGs; 1098 DEGs belonged only to the Fries 3 vs PD group and 773 DEGs belonged only to the PS vs PD group. Comparison of the DEGs from the Fries3 vs PD group and from the Fries3 vs PS group revealed 557 common DEGs; 2043 DEGs belonged only to the Fries3 vs PD group and 301 DEGs belonged only to the Fries3 vs PS group. Comparison of the DEGs from the Fries3 vs PS group and PS vs PD group revealed 405 common DEGs; 471 DEGs belonged only to the Fries3 vs PS group and 1888 DEGs belonged only to the PS vs PD group. Comparison of DEGs in all three groups revealed 208 DEGs common between the three groups (Figure 4B). The heatmap showed that each group had some highly expressed genes, whereas the expression profiles of Fries 3 and PS were similar (Figure $4 C)$.

\section{GO analysis of DEGs}

The unigenes were compared with the GO database by BLAST, and the DEGs were analyzed for GO enrichment. The numbers of DEGs in the Fries3 vs PD group enriched for BP, MF, and CC were 735, 1011, and 0 , respectively. The DEGs enriched in BP were mainly concentrated in three subcategories including oxidation-reduction process $(383,10.51 \%)$, transmembrane transport $(249,13.34 \%)$, and monocarboxylic acid metabolic process $(103,5.52 \%)$. The DEGs enriched in MF were mainly concentrated in 6 subcategories including oxidoreductase activity (394, 21.1\%); coenzyme binding, cofactor binding (182, 9.75\%); flavin adenine dinucleotide binding (74,3.96\%); oxidoreductase activity, acting on $\mathrm{CH}-\mathrm{OH}$ group of donors $(89,4.77 \%)$; and oxidoreductase activity, acting on paired donors, with incorporation or reduction of molecular oxygen $(66,3.54 \%$ ) (Figure $5 A$ ). The number of DEGs in the Fries3 vs and PS group enriched for $\mathrm{BP}, \mathrm{MF}$, and CC were 158,762 , and 0 , respectively. DEGs enriched in BP were mainly concentrated in the subcategories of oxidation-reduction process $(158,25.44 \%)$. DEGs enriched in MF were mainly concentrated in four subcategories including oxidoreductase activity $(167,26.89 \%)$, coenzyme binding $(90,14.49 \%)$, cofactor binding $(93,14.98 \%)$, and catalytic activity $(412,66.34 \%)$ (Figure 5B). The numbers of DEGs in the PS vs PD group enriched for BP, MF, and CC were 236, 681, and 0, respectively. DEGs enriched in BP were mainly concentrated in the subcategories of transmembrane transport $(236,14.07 \%)$. DEGs enriched in MF were mainly concentrated 3 subcategories including oxidoreductase activity (338, $20.16 \%)$, coenzyme binding (162, 9.66\%), and cofactor binding (181, 10.79\%) (Figure 5C).

\section{KEGG analysis of DEGs}

The DEGs were searched in the KEGG database to identify physiological processes in which they might participate. The DEGs in the Fries3 vs PD group were enriched in metabolic pathways such as betaalanine metabolism, amino sugar and nucleotide sugar metabolism, propanoate metabolism, tyrosine metabolism, sphingolipid metabolism, tryptophan metabolism, glycerolipid metabolism, starch and 
sucrose metabolism, glycerophospholipid metabolism, arachidonic acid metabolism, and phenylalanine metabolism. They were also enriched in biosynthesis pathways such as various types of $\mathrm{N}$-glycan biosynthesis and fatty acid biosynthesis (Figure 6A). DEGs in the Fries3 vs PS group were enriched in several pathways such as amino sugar and nucleotide sugar metabolism, sulfur metabolism, propanoate metabolism, glycolysis/gluconeogenesis, methane metabolism, fatty acid degradation, pentose and glucuronate interconversions, nicotinate and nicotinamide metabolism, beta-alanine metabolism, starch and sucrose metabolism, glycerophospholipid metabolism, regulation of autophagy, peroxisome, phagosome, pentose phosphate pathway, glutathione metabolism, glyoxylate and dicarboxylate metabolism, cyanoamino acid metabolism, folate biosynthesis, and homologous recombination (Figure $6 B$ ). DEGs in the PS vs PD group were enriched in many pathways associated with ribosomes, betaalanine metabolism, peroxisomes, amino sugar and nucleotide sugar metabolism, sphingolipid metabolism, propanoate metabolism, various types of $\mathrm{N}$-glycan biosynthesis, ubiquitin-mediated proteolysis, valine, leucine and isoleucine degradation, regulation of autophagy, glycerophospholipid metabolism, glutathione metabolism, $\mathrm{N}$-glycan biosynthesis, steroid biosynthesis, MAPK signaling pathway - yeast, vitamin B6 metabolism, one carbon pool by folate, thiamine metabolism, sulfur relay system, and phenylalanine metabolism (Figure 6C). Pathways common between the top 20 DEGs' of the 3 groups were beta-alanine metabolism, peroxisome, amino sugar and nucleotide sugar metabolism, propanoate metabolism, and glycerophospholipid metabolism.

\section{CAZyme analysis of DEGs}

By comparing the protein sequences using the CAZymes analysis toolkit software package, 352 CAZyme families with 325 genes were annotated. Among them, there were $39(11 \%)$ carbohydrate binding modules (CBM), 61 (17\%) carbohydrate esterase (CE), 140 (40\%) glycoside hydrolases (GH), 71 (20\%) glycosyl transferases (GT), and 41 (12\%) auxiliary activities (AA) (Figure 7A). Within CBM, CE, GH, GT, and AA, there were $18,12,49,28$, and 8 gene families, respectively (Supplementary Table S3).

The numbers of CAZymes in DEGs of the Fries3 vs PD group, PS vs PD group, and Fries3 vs PS group were 134, 97, and 46, respectively. Within the 134 CAZymes in DEGs of the Fries3 vs PD group, there were 22 AAs (13 upregulated and 9 downregulated), 18 CBMs (12 upregulated and 6 downregulated), 15 CEs (10 upregulated and 5 downregulated), $61 \mathrm{GHs}$ (33 upregulated and 27 downregulated), and $18 \mathrm{GTs}$ (7 upregulated and 11 downregulated). Interestingly, 10 were not expressed in the PD group but were highly expressed in the Fries3 group (Figure 7B). Within the 97 CAZymes in DEGs of the PS vs PD group, there were 13 AAs (10 up and 3 down), 11 CBMs ( 7 up and 4 down), 11 CEs (5 up and 6 down), 48 GHs (25 up and 23 down), and 14 GTs ( 6 up and 8 down). Interestingly, 6 were not expressed in the PS group but were highly expressed in the PD group (Figure 7C). Within the 46 CAZymes in DEGs of the Fries 3 vs PS group, there were 10 AAs ( 3 up and 7 down), 8 CBMs (4 up and 4 down), 5 CEs (2 up and 3 down), 22 GHs (9 up and 13 down), and 1 GT (up) (Figure 7D).

\section{Discussion}


Under different fermentation conditions, the biological control strains show varied effects, including variability in pathogen control. Zhuang et al. [25] reported that the Trichoderma strain 23 produced chlamydospores better in the PD and PS liquid media than in eight other tested liquid media. Further, strain 23 showed the highest hyphal quantity and chitinase activity in PDB medium (containing $20 \%$ potato juice and $2 \%$ sucrose, same as PS in this study). In the present study, the T. koningiopsis Tk1 cultured with PS and Fries3 media showed stronger pathogen inhibition effects. The differences in chitinase activities of the Tk1 strain in the two media may explain the difference in percentage inhibition between these media. Identification of the KEGG pathways enriched in the DEGs highlighted the differences in some metabolism pathways enriched under the three different conditions; further, it revealed that the differences in produced metabolic products may have caused differences in pathogen/disease control effects. The differences in metabolic products leading to differences in biocontrol require further analysis.

Some exogenous substances, such as the source of carbon, may induce fungi to produce different important metabolites. Xiang [26] reported that Nimbya alternantherae produced a toxin against Alternanthera philoxeroides in modified Fries3 medium but not in potato carrot medium. Vulculic acid was the only major compound of $\mathrm{N}$. alternantherae in Fries3 medium, as compared to in potato carrot medium, as detected by the largest peak in the chromatograph [27]. Sucrose is a good source of carbon for the mass production of Trichoderma [28]. Ike and Tokuyasu [29] showed that sucrose is a good carbon source for T. reesei M2-1 for the enzyme production system. Maibam and Tokuyasu [30] demonstrated that filter-paperase and carboxymethyl cellulase produced by $T$. reesei were increased by five-fold upon induction with sugarcane bagasse. We observed similar results in our study, as $T$. koningiopsis Tk1 cultured in Fries3 and PS had better antimicrobial effects; both Fries3 and PS media contained sucrose. Thus, sucrose was the best carbon source for T. koningiopsis Tk1 for biocontrol against $F$. oxysporum. However, T. koningiopsis Tk1 showed good mycelial growth and sporulation in all three liquid media. Therefore, sucrose may play an important role in the production of $T$. koningiopsis Tk1 enzymes and metabolites with an antimicrobial effect.

Degradation of the cell wall components of pathogenic fungi by secreting cell wall-degrading enzymes that include chitinase, glucanase, cellulase, xylanase, pectinase, and protease among others is an effective antagonistic method [31]. Some cell wall-degrading enzymes produced by biocontrol fungi can promote the adsorption, winding, invasion, digestion, and other functions, of the acceptor fungus against the pathogen to control pathogen growth and reproduction. Trichoderma enzyme-encoding genes have been used to improve plant resistance to pathogens [32,33]. However, none of these transgenic cultivars have been commercialized. In our study, some genes encoding CAZymes were differentially expressed between the three different culture conditions. There were fewer differentially expressed CAZymes in Fries3 and PS, both of which showed a similar pathogen inhibition effect. Thus, the differentially expressed CAZymes may be important because of their ability to cause pathogen inhibition differently in different media. Therefore, all differentially expressed CAZymes identified in this study should be further explored and validated. 
This is the first report of the transcriptome of $T$. koningiopsis and the differences in the transcriptome under varying conditions. The DEGs should be further examined to confirm their functions and the interaction mechanism between gene.

\section{Conclusion}

The present study revealed that the mycelium weight and sporulation of $T$. koningiopsis Tk 1 differed in PS, PD, and Fires 3 media. The Tk1 strain inhibited growth of the pathogen Fusarium oxysporum in the three media tested. Fries3, PD, and PS were collected for RNA sequencing of the Tk1 mycelia grown on three different media to identify DEGs between Tk1 grown on different media. De novo transcriptome assembly revealed 14,208 unigenes. The DEG pattern was more similar between Fries 3 and PS samples, showing very different expression patterns compared to PD samples. The DEGs were enriched in some metabolic and biosynthetic pathways. Additional DEG analysis identified a set of carbohydrate-active enzymes upregulated or downregulated under different conditions. The results indicate that the Tk1 strain cultured in Fires3 and PS mediums produces specific components, including metabolic and CAZymes that enhance their antimicrobial effect, providing a foundation for subsequent mining of specific genes. The DEGs should be further evaluated to confirm their function and interaction mechanisms.

\section{Methods}

\section{Strains and culture media}

The T. koningiopsis Tk1 strain used in this study was isolated from a medicinal plant, Chrysanthemum morifolium "Huangju" and maintained at the Zhongkai University of Agriculture and Engineering, China. The root wilt pathogen F. oxysporum [34] was obtained from the Innovative Institute for Plant Health, Zhongkai University of Agriculture and Engineering. The stock cultures were stored at $4{ }^{\circ} \mathrm{C}$ on potato dextrose agar (PDA) slants. Fungi were grown on PDA plates at $28^{\circ} \mathrm{C}$ for $2-7$ days.

The PS medium ( $1000 \mathrm{~mL}$ ) was composed of $200 \mathrm{~g}$ boiled and peeled potatoes, $20 \mathrm{~g}$ sucrose, and distilled water to make up the final volume. The PD medium $(1000 \mathrm{~mL})$ was composed of $200 \mathrm{~g}$ peeled potatoes boiled in water, glucose $20 \mathrm{~g}$, and distilled water to make up the final volume. The Fries medium no. 3 (Fries3) (1000 mL) was composed of $20 \mathrm{~g}$ sucrose, $5 \mathrm{~g}$ tartaric acid, $1 \mathrm{~g} \mathrm{NH}_{4} \mathrm{NO}_{3}, 1 \mathrm{~g} \mathrm{KH}_{2} \mathrm{PO}_{4}, 0.5 \mathrm{~g}$ $\mathrm{MgSO}_{4} .7 \mathrm{H}_{2} \mathrm{O}, 0.13 \mathrm{~g} \mathrm{CaCl}_{2}, 0.5 \mathrm{~g}$ yeast extract, and distilled water to make up the final volume. The preculture media were sterilized at $121^{\circ} \mathrm{C}$ for $20 \mathrm{~min}$.

\section{Mycelium growth andsporulation of $T$. koningiopsis Tk1 strain in different media}

A 5-mm mycelium was added into the PD medium and incubated on a rotary shaker (180 rpm) for $24 \mathrm{~h}$. Next, $1 \mathrm{~mL}$ suspension was added into the PS, PD, and Fries3 medium and incubated on the rotary shaker (180 rpm) for $7 \mathrm{~d}$. Three replicates were cultured in each medium. After $7 \mathrm{~d}$, the suspensions were filtered with sterile gauze, followed by collection of the mycelia into a culture dish. The collected mycelia were 
baked for $24 \mathrm{~h}$ at $55^{\circ} \mathrm{C}$ in an oven and weighed. The filtrates were checked for sporulation by counting spores using a hemocytometer.

\section{Filtrate antifungal bioassay}

The filtrates were again filtered using $0.22-\mu \mathrm{m}$ polyethersulfone filters. A volume of $3 \mathrm{~mL}$ aseptic filtrate was added to $7 \mathrm{~mL}$ PDA and mixed at approximately $55^{\circ} \mathrm{C}$. Each group of the PD, PS, and Fries 3 had three replicates. Next, a 5-mm mycelial disc of the pathogen F. oxysporum was placed in the middle of the plates, and medium was added to the PDA plate; PDA alone was used as a control. The plates were incubated at $28^{\circ} \mathrm{C}$ for $7 \mathrm{~d}$, followed by measurement of pathogen colony diameters; the experiments were repeated twice. The colony diameter of F. oxysporum was measured and converted to percentage inhibition using the following formula:

Percentage inhibition of $F$. oxysporum diameter $(\%)=$,

where Dc and Dt are the diameters of the pathogen in the untreated control and treatment group, respectively [35].

\section{RNA extraction and transcriptome sequencing}

The mycelial samples from the PS, PD, and Fries 3 conditions were extracted after $7 \mathrm{~d}$ of fermentation. Mycelia were collected by centrifugation at $12,000 \times g$ and $4^{\circ} \mathrm{C}$ and stored at $-80^{\circ} \mathrm{C}$, followed by RNA extraction using RNeasy Plus Universal Kits (Qiagen, Hilden, Germany).

RNA purity was measured using a NanoPhotometer ${ }^{\circledR}$ spectrophotometer (IMPLEN, Munich, Germany). RNA integrity was assessed using the RNA Nano 6000 Assay Kit of the Agilent Bioanalyzer 2100 system (Agilent Technologies, Santa Clara, CA, USA).

Libraries were constructed using $1.5 \mu \mathrm{g}$ of each RNA sample and the NEBNext ${ }^{\circledR}$ Ultra ${ }^{\text {TM }}$ RNA Library Prep Kit for Illumina ${ }^{\circledR}$ (New England Biolabs, Ipswich, MA, USA) according to the manufacturer's instructions. Polymerase chain reaction products were purified (AMPure XP system) and library quality was assessed on the Agilent Bioanalyzer 2100 system. Clustering of index-coded samples was performed on a cBot Cluster Generation System using TruSeq PE Cluster Kit v3-cBot-HS (Illumina, San Diego, CA, USA) according to the manufacturer's instructions. The libraries were classified based on the preculture media of the samples: "PS" library, "PD" library, and "Fries3" library. Paired-end sequencing was performed on an Illumina HiSeq platform to generate reads.

\section{Sequencing data processing and analysis}

Raw data (raw reads) in the FASTQ format were processed using in-house Perl scripts. Clean reads were obtained by trimming raw reads to remove adapter sequences, poly- $\mathrm{N}$, and low-quality bases from the raw data, and quality scores (Q20, Q30), GC-content, and the sequence duplication level of the processed data were calculated. All downstream analyses were performed on high-quality processed data. Transcriptome 
assembly was accomplished using FASTQ files for paired-end reads, left.fq and right.fq, using Trinity [36] with the parameter, min_kmer_cov, set to 2 (default) and all other parameters set to default values. The resultant contigs were compared with the following databases using BLAST to identify homologous sequences: NCBI RefSeq (non-redundant protein database, NR, and non-redundant nucleotide sequences, $\mathrm{Nt}$ ), Pfam (Protein family), KOG/COG (Clusters of Orthologous Groups of proteins), Swiss-Prot (manually annotated and reviewed protein sequence database), KO (KEGG Ortholog database), and GO (Gene Ontology).

Differential expression analysis of each pair of conditions was performed using the DESeq R package (1.10.1). The resulting $P$ values were adjusted using the Benjamini-Hochberg approach for controlling the false discovery rate. Genes found by DESeq with an adjusted P-value $<0.05$ were designated as differentially expressed. GO enrichment analysis of the DEGs was implemented by the GOseq R packagebased Wallenius' noncentral hypergeometric distribution [37]. KOBAS [38] software was used to test the statistical enrichment of DEGs in the KEGG pathways. Further, the DEGs in each group were aligned against the T. koningiopsis Tk1 strain's CAZyme transcriptome.

\section{Data availability}

The data generated from RNA sequencing were submitted to NCBI under accession number SUB7785630. The raw sequences (archives of paired-end sequences) generated from the PS (SAMN15581379 and SAMN15581380), Fries3 (SAMN15581381 and SAMN15581382), and PD libraries (SAMN15581383 and SAMN15581384) were submitted to the NCBI Sequence Read Archive.

\section{Statistical analyses}

The data were analyzed using Data Processing System (Hangzhou, China). Normality and homoscedasticity were evaluated. All data were analyzed by analysis of variance followed by Fisher test $(P<0.05)$.

\section{Declarations}

\section{Ethics approval and consent to participate}

Not Applicable.

\section{Consent for publication}

Not Applicable.

\section{Availability of data and materials}

All data sets analyzed here are publicly available. The data generated from RNA sequencing in our study were submitted to NCBI under accession number SUB7785630. The raw sequences (archives of pairedend sequences) generated from the PS (SAMN15581379 and SAMN15581380), Fries3 (SAMN15581381 
and SAMN15581382), and PD libraries (SAMN15581383 and SAMN15581384) were submitted to the NCBI Sequence Read Archive independently.

\section{Competing interests}

The authors declare that they have no competing interests.

\section{Funding}

This project was funded by the grant from Key Realm R\&D Program of Guangdong Province (grant number "2018B020205003"), Characteristic innovation projects of general colleges and universities in Guangdong Province (grant number "2018KTSCX097") and Guangdong science and technology special commissioner project. The funding agency played no role in the design of the study and collection, analysis, and interpretation of data and in writing the manuscript.

\section{Authors' contributions}

$M L$ and $Z Y D$ designed the project. $M L, Y X S$, and $M B C$ conducted the experiments and prepared the samples. ML, YXS, and ZYD analysed the data. ML, and ZYD wrote the paper. All authors read and approved the final manuscript.

\section{Acknowledgements}

We would like to thank Department of science and technology of Guangdong Province for funding the Key Realm R\&D Program of Guangdong Province (2018B020205003), Guangdong science and technology special commissioner project, department of education of Guangdong Province for funding Characteristic innovation projects of general colleges and universities in Guangdong Province (2018KTSCX097).

\section{Author details}

Innovative Institute for Plant Health, Zhongkai University of Agriculture and Engineering, Guangzhou 510225, PR of China

\section{References}

1. Chen JL, Liu K, Miao CP, Sun SZ, Chen YW, Xu LH, Guan HL, Zhao LX. Salt tolerance of endophytic Trichoderma koningiopsis yim ph30002 and its volatile organic compounds (vocs) allelopathic activity against phytopathogens associated with panax notoginseng. Ann Microbiol. 2016;66(3):981-90.

2. Zhang Y, Zhuang WY. Trichoderma brevicrassum strain TC967 with capacities of diminishing cucumber disease caused by Rhizoctonia solani and promoting plant growth. Biol Control. 2020;142:104151. 
3. Bissett J, Gams W, Jaklitsch W, Samuels GJ. Accepted Trichoderma names in the year 2015. Ima Fungus. 2015;6(2):263-95.

4. Shi XS, W DJ, Li XM, Li HL, Meng LH, Li X. Antimicrobial polyketides from Trichoderma koningiopsis qa-3, an endophytic fungus obtained from the medicinal plant artemisia argyi. Rsc Advances. 2017;7(81):51335-42.

5. Tandon A, Fatima T, Anshu Yadav U, Srivastava S, Singh PC. Effect of Trichoderma koningiopsis on Chickpea Rhizosphere Activities under Different Fertilization Regimes. Open Journal of Soil Science. 2018;8:261-75.

6. Delgado-Jarana J, Moreno-Mateos MA, Benítez T. Glucose uptake in Trichoderma harzianum: role of gtt1. Euk Cell. 2003; (2): 708-717.

7. Bailey BA, Strem MD, Wood D. Trichoderma species form endophytic associations within Theobroma cacao trichomes. Mycol Res. 2009;113(12):1365-76.

8. Vos CMF, De Cremer K, Cammue BPA, De Coninck B. The toolbox of Trichoderma spp. in the biocontrol of Botrytis cinerea disease. Mol Plant Pathol. 2015;16(4):400-12.

9. Sharma V, Bhandari P, Bikram S. Chitinase expression due to reduction in fusaric acid level in an antagonis Tictrichoderma harzianum S17TH. Indian J Microbiol. 2013;53(2):214-20.

10. Chinheya CC, Yobo KS, Laing MD. Biological control of the root knot nematode, Meloidogyne javanica, (chitwood) using, bacillus, isolates, on soybean. Biol Control. 2017;109:37-41.

11. Shang J, Liu B, Xu Z. Efficacy of Trichoderma asperellum TC01 against anthracnose and growth promotion of Camellia sinensis seedlings, Biol Control. (2020).

12. Lorito M, Farkas V, Rebuffat $S$, Bodo B, Kubicek CP. Cell wall synthesis is a major target of mycoparasitic antagonism by Trichoderma harzianum. J Bacteriol. 1996;178(21):6382-5.

13. da Silva JAT, de Medeiros EV, da Silva JM, de AD, Moreira, da Dilva Nascimento KA, Souza-Motta TCE. C. Trichoderma aureoviride URM 5158 and Trichoderma hamatum URM 6656 are biocontrol agents that act against cassava root rot through different mechanisms. J Phytopathol. 2016;164(11-12):1003-11.

14. Weindling R. Studies on a lethal principle effective in the parasitic action of Trichoderma lignorum on Rhizoctonia solani and other soil fungi. Phytopathology. 1934;24:1153-79.

15. SUÁREZ YYJ, Velndia CAM, Prado AMC. Induced systemic resistance against Fusarium oxysporum in tomato by Trichoderma koningiopsis Th003. Aata Biol Colomb. 2009;14(3):111-20.

16. Liu K, Yang YB, Chen JL, Miao CP, Wang Q, Zhou H, Chen YW, Li YQ, Ding ZT, Zhao LX. Koninginins nq, polyketides from the endophytic fungus Trichoderma koningiopsis harbored in panax notoginseng. Natural Products Bioprospecting. 2016;6:49-55.

17. Arinbasarova AY, Baskunov BP, Medentsev AG. A low-molecular mass antimicrobial peptide from Trichoderma aureoviride Rifai VKM F-4268D. Microbiology 2017;86(2):289-291.

18. Bisen K, Keswani C, Patel JS, Sarma BK, Singh HB. Trichoderma spp.: Efficient Inducers of Systemic Resistance in Plants. In: Choudhary D, Varma A, editors Microbial-mediated Induced Systemic 
Resistance in Plants. 2016.

19. Collins RP, Halim AF. Characterization of the Major Aroma Constituent of the Fungus Trichoderma viride (Pers.). J Agr Food Chem. 1972;20(2):437-8.

20. Claydon N, Allan M, Hanson JR, Avent AG. Antifungal alkyl pyrones of Trichoderma harzianum. Transactions of the British Mycological Society. 1987;88(4):503-13.

21. Dunlop RW, Simon A, Sivasithamparam K, Ghisalberti EL. An antibiotic from Trichoderma koningii active against soilborne plant pathogens. J Nat Prod. 1989;52(1):67-74.

22. Jeleń H, Błaszczyk L, Chełkowski J, Rogowicz K, Strakowska J. Formation of 6-n-pentyl-2 h-pyran-2one (6-pap) and other volatiles by different Trichoderma species. Mycol Prog. 2014;13(3):589-600.

23. Horta MAC, Vicentini R, Delabona PDS, Laborda P, Crucello A, Freitas S, Kuroshu RM, Polikarpov I, da Cruz Pradella JG, Souza AP. Transcriptome Profile of Trichoderma harzianum IOC-3844 Induced by Sugarcane Bagasse. PLoS One. 2014;9(2):e88689.

24. Bech L, Busk PK, Lange L. Cell wall degrading enzymes in Trichoderma asperellum grown on wheat bran. Fungal Genomics \& Biology, 2015; 4(1): [1000116].

25. Zhuang JH, Gao ZG, Liu X, Chen J, Yang Y, Huang YQ. Effect of fermentation factors on spore types of Trichoderma strain 23. Chinese Journal of Biological control. 2005;21(1):37-40.

26. Xiang MM, Huang PG, Jiang ZH, Xue Lian OU, Wang LH. Preliminary Study of Phytotoxic Metabolites with Herbicidal Activity Produced by Nimbya alternantherae. Journal of Yunnan Agricultural University. 2002;17:352-5.

27. Xiang M, Chen S, Wang L, Dong Z, Huang J, Zhang Y, Strasser RJ. Effect of vulculic acid produced by Nimbya alternantherae on the photosynthetic apparatus of Alternanthera philoxeroides. Plant Physiol Biochem. 2013;65:81-8.

28. Ghazanfar MU, Raza M, Raza W. Effect of physiological parameters on mass production of Trichoderma species. Pakistan Journal of Phytopathology. 2018;30(1):59-65.

29. Ike M, Tokuyasu K. Cellulase production of Trichoderma reesei (hypocrea jecorina) by continuously fed cultivation using sucrose as primary carbon source. J Appl Glycosci. 2018;65(4):51-6.

30. Maibam PD, Maiti SK. A strategy for simultaneous xylose utilization and enhancement of cellulase enzyme production by Trichoderma reesei cultivated on liquid hydrolysate followed by induction with feeding of solid sugarcane bagasse. Waste \& Biomass Valorization. 2019.

31. Harman GE, Howell CR, Viterbo A, Chel I, Lorito M. Trichoderma species-opportunistic, avirulent plant symbionts. Nat Rev Microbiol. 2004;2(1):43-56.

32. Lorito M, Woo SL, Garcia I, Colucci G, Harman GE, Pintor-Toro JA, Filippone E, Muccifora S, Lawrence CB, Zoina A, Tuzun S, Scala F, Fernandz IG. Genes from mycoparasitic fungi as a source for improving plant resistance to fungal pathogens. Proc. Natl. Acad. Sci. USA 1998;95: 7860-7865.

33. Bolar JP, Norelli JL, Wong KW, Hayes CK, Harman GE, Aldwinckle HS. Expression of endo-chitinase from Trichoderma harzianum in transgenic apple increases resistance to apple scab and reduces vigor. Phytopathology. 2000;90:72-7. 
34. Chen XY, Shu YX, Luo M, Xiang MM, Huang YH, Zhang WL, Dong ZY. Fusarium Wilt of Imperial Chrysanthemum (Chrysanthemum morifolium) Caused by Fusarium oxysporum in China. Plant dis. 2020;104(3):985.

35. Prapagdee B, Akrapikullchart L, Mongkolsuk S. Potential of a soil-borne streptomyces hygroscopicus for biocontrol of an anthracnose disease caused by Colletotrichum gloeosporioides in orchid. Journal of biological sciences, 2008;8(7).

36. Grabherr MG, Haas BJ, Yassour M, Levin JZ, Thompson DA, Amit I, Adiconis X, Fan L, Raychowdhury R, Zeng QD, Chen ZH, Mauceli E, Nusbaum C, Lindblad-Toh K, Friedman N, Regev A. Full-length transcriptome assembly from RNA-Seq data without a reference genome. Nat Biotechnol. 2011;29:644-52. (Trinity).

37. Young MD, Wakefield MJ, Smyth GK, Oshlack A. Gene ontology analysis for RNA-seq: accounting for selection bias. Genome Biol. 2010; (GOseq).

38. Mao X, Cai T, Olyarchuk JG, Wei LP. Automated genome annotation and pathway identification using the KEGG Orthology (KO) as a controlled vocabulary. Bioinformatics. 2005;21:3787-93. (KOBAS).

\section{Figures}

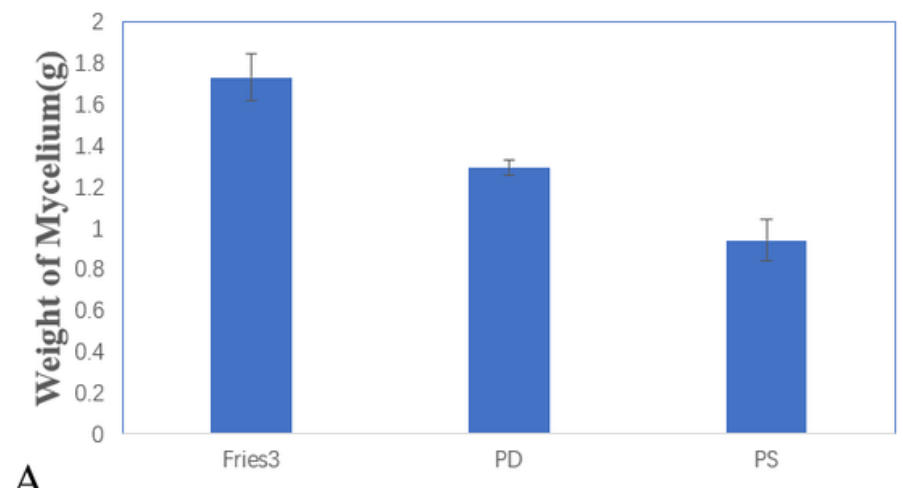

A

Different culture medium

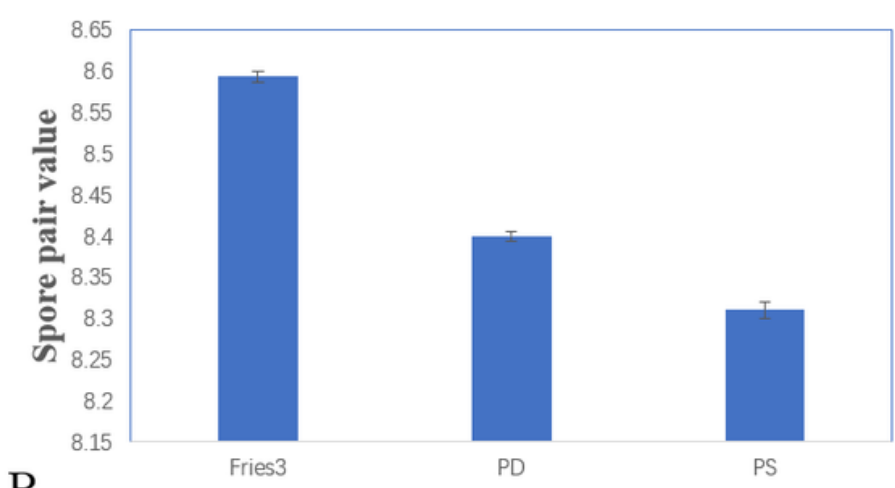

B Different culture medium

\section{Figure 1}

The mycelium growth and sporulation of Trichoderma koningiopsis Tk1 strain in different medium (A) The mycelium growth of T. koningiopsis Tk1 strain in different medium (B) The sporulation of T. koningiopsis Tk1 strain in different medium 


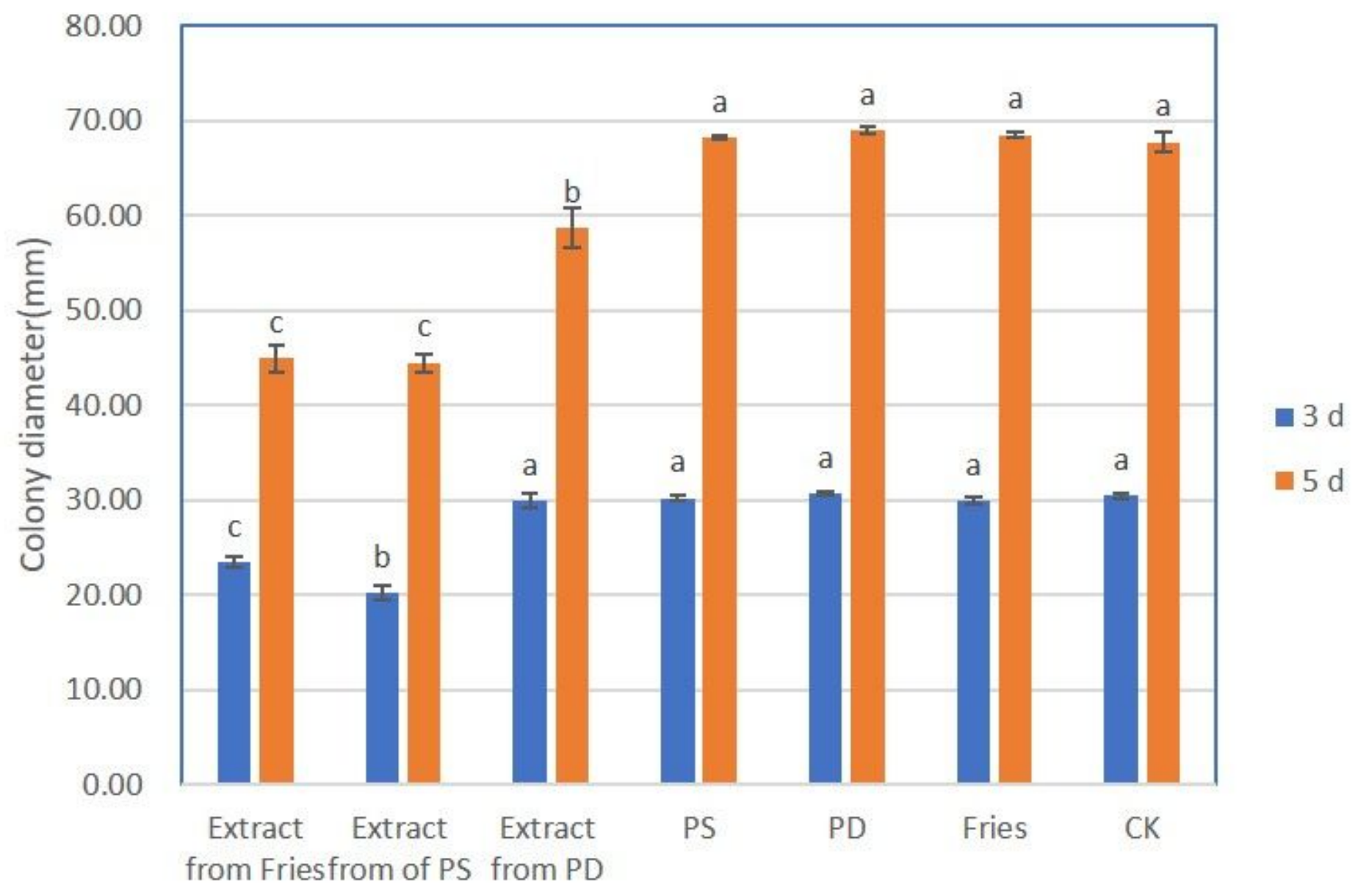

Figure 2

The filtrate antifungal bioassay of Trichoderma koningiopsis Tk1 strain in different medium against Fusarium oxysporum 

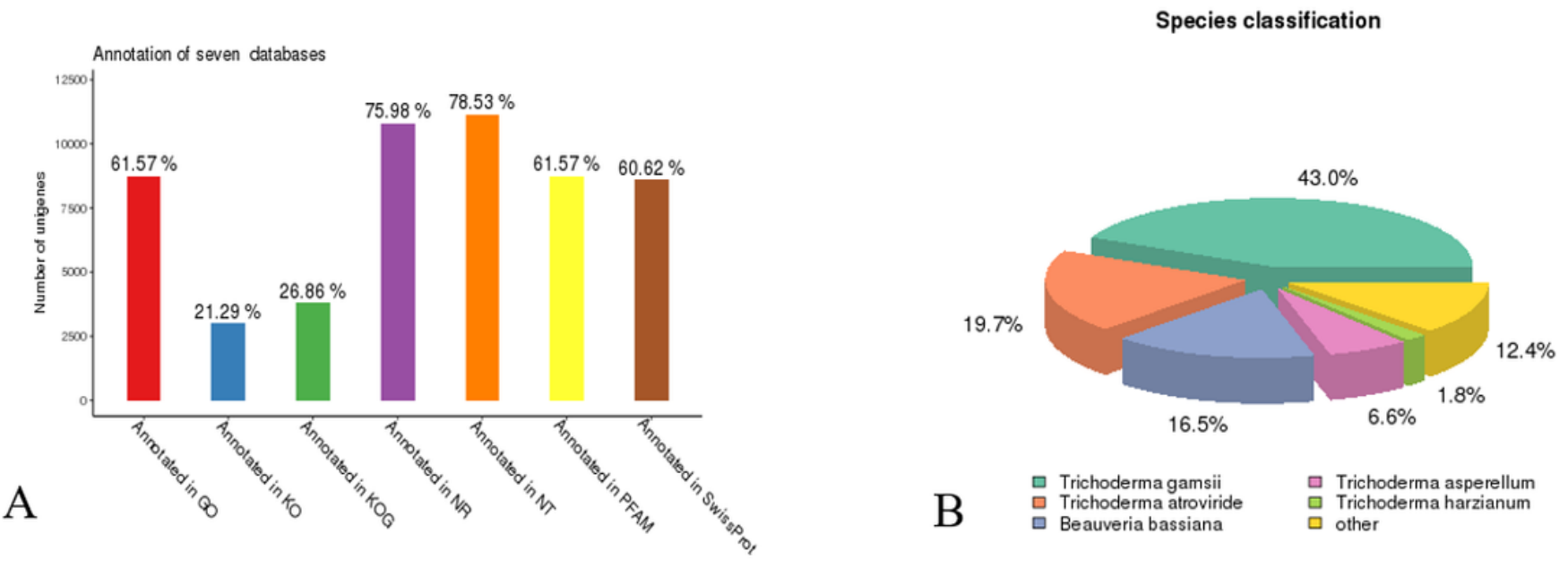

Gene Function Classification (GO)

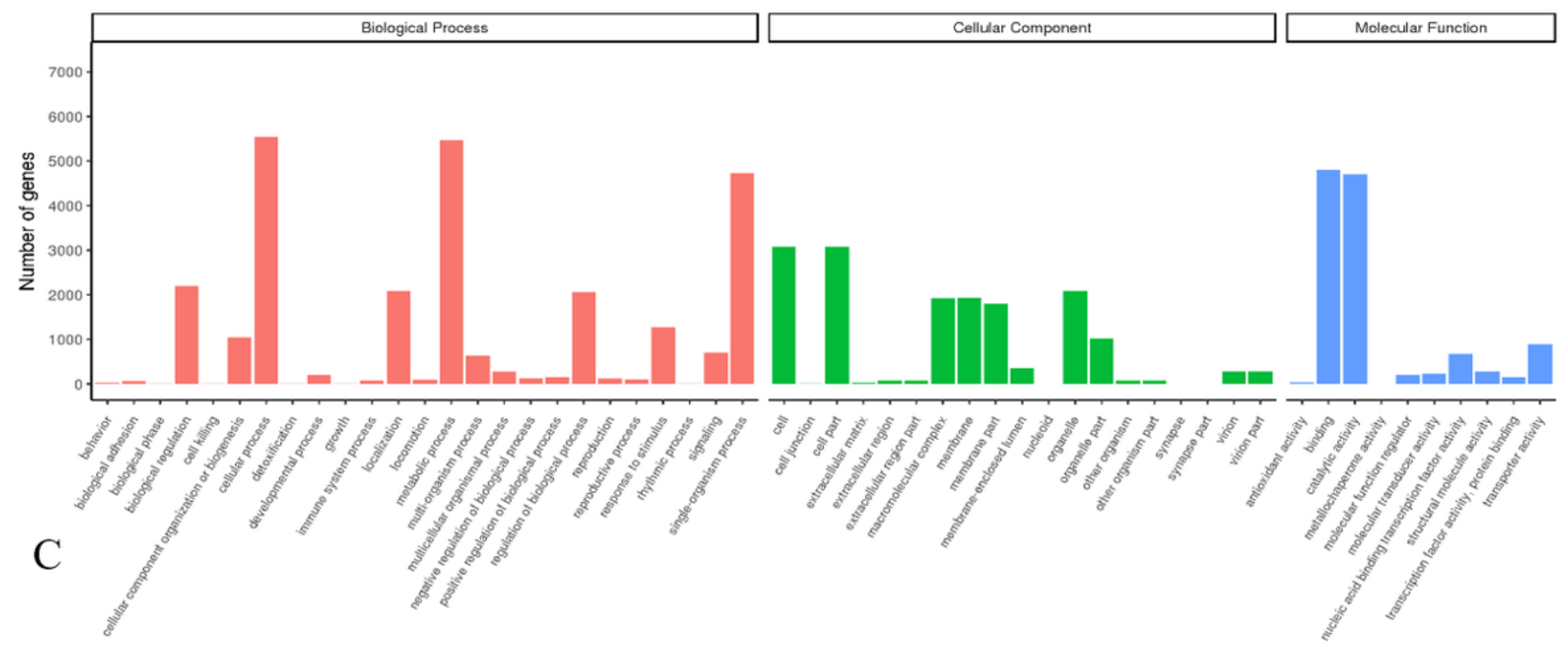

Figure 3

(A) Unigenes annotated via different databases. (B) Percentage of top hits from other Fungi species homologous to Trichoderma koningiopsis transcripts. (C) Gene ontology (GO) classification of Trichoderma koningiopsis transcripts using Blast2GO. 


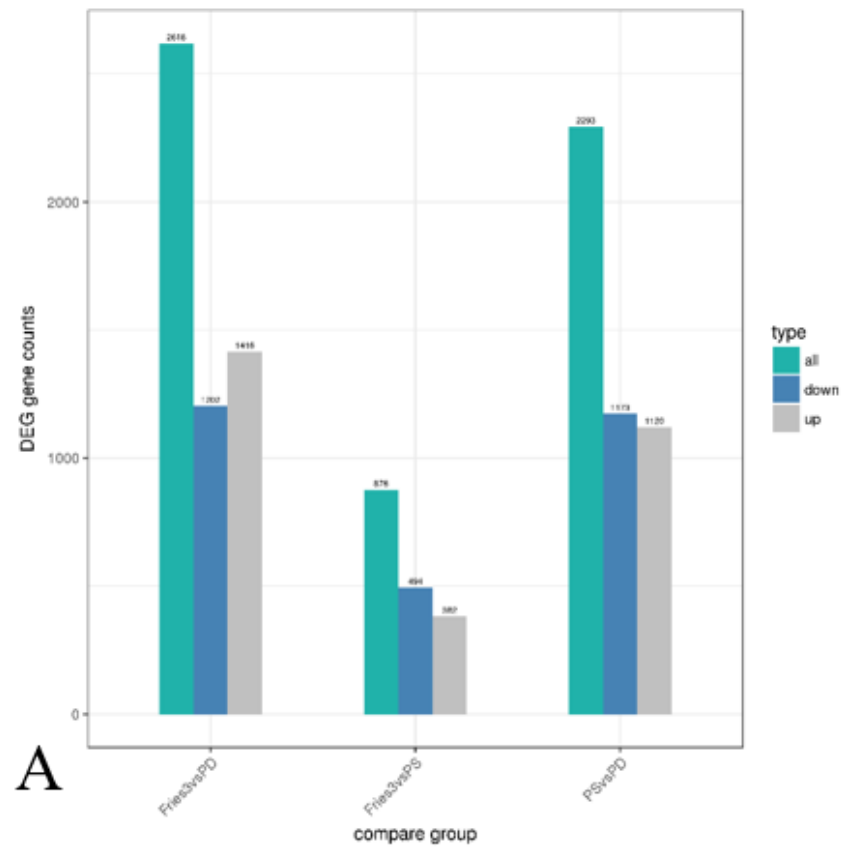

Cluster analysis of differentially expressed genes

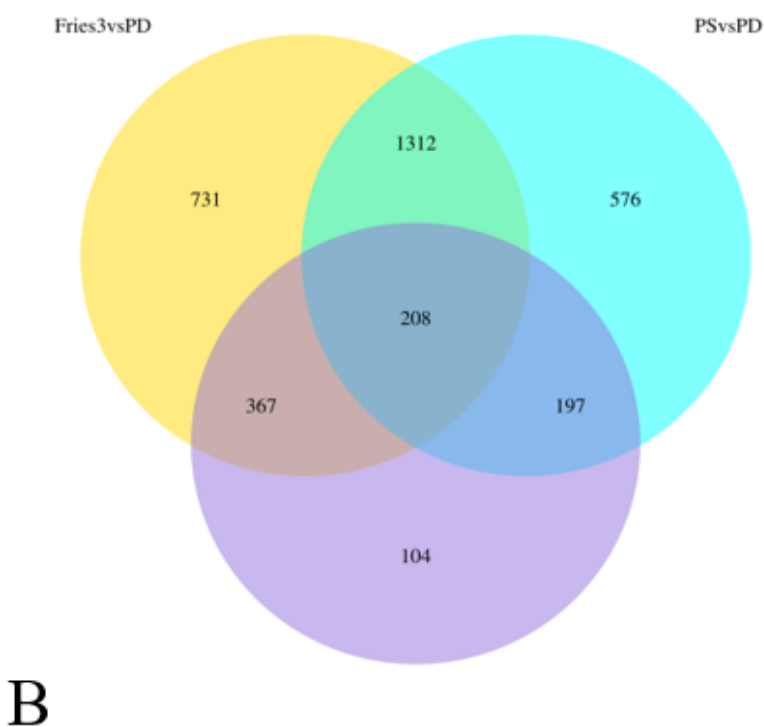

Fries3vsPS

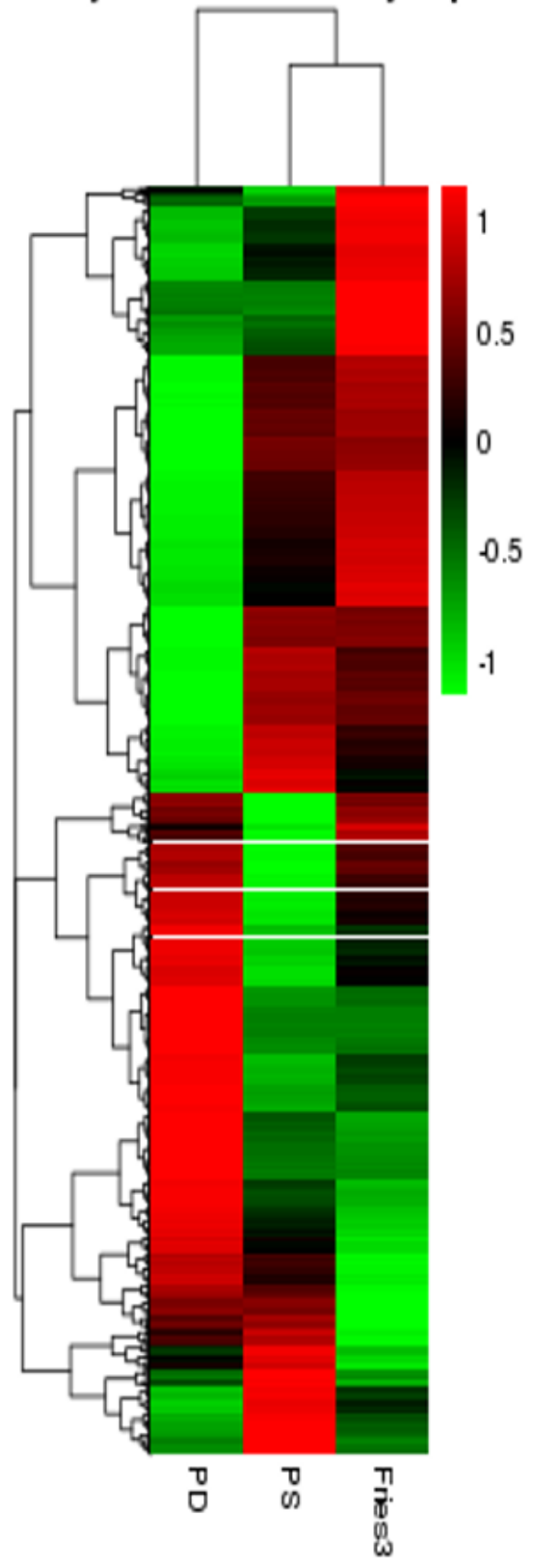

Figure 4

Analysis of differential expression genes of Trichoderma koningiopsis under Fries, PS and PD liquid mediums. (A) The numbers of differential expression genes. (B) The Venn analysis of differential expression genes. (C) The heatmap of differential expression genes. 

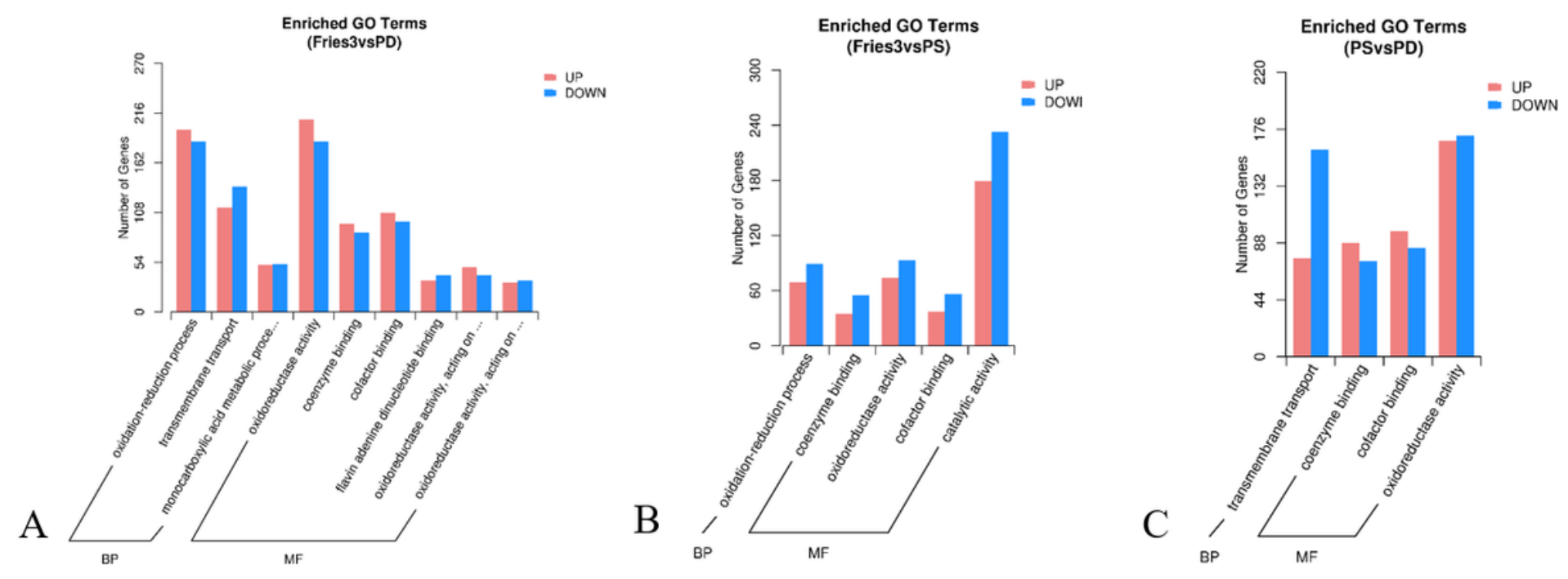

\section{Figure 5}

The GO analysis in the differential expression genes. (A) The GO analysis in the differential expression genes between Fries3 and PD. (B) The GO analysis in the differential expression genes between Fries3 and PS. (C) The GO analysis in the differential expression genes between PS and PD.
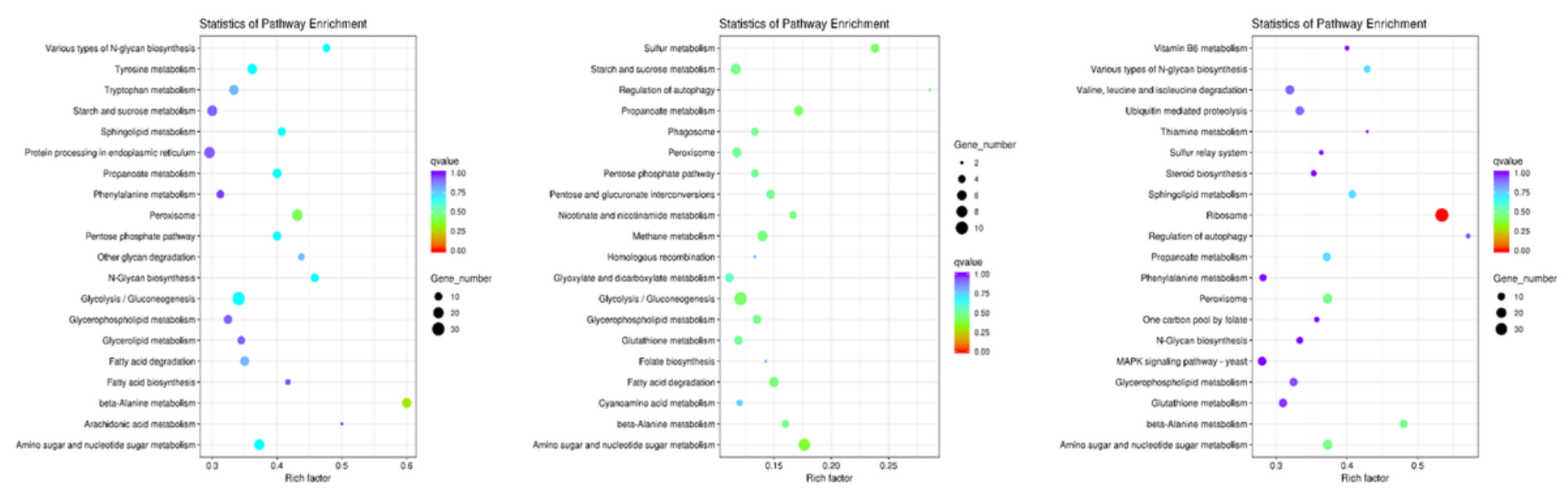

Figure 6

The KEGG analysis in the differential expression genes. (A) The KEGG analysis in the differential expression genes between Fries3 and PD. (B) The KEGG analysis in the differential expression genes between Fries3 and PS. (C) The KEGG analysis in the differential expression genes between PS and PD. 


\section{Summary of the six CAZyme categories}

A

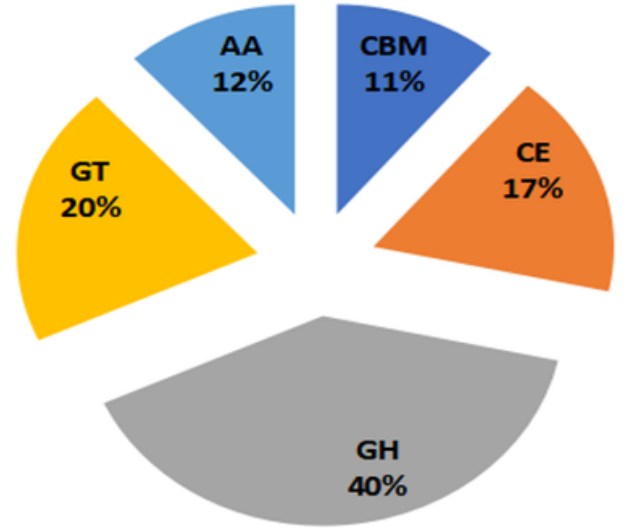

PSvsPD group

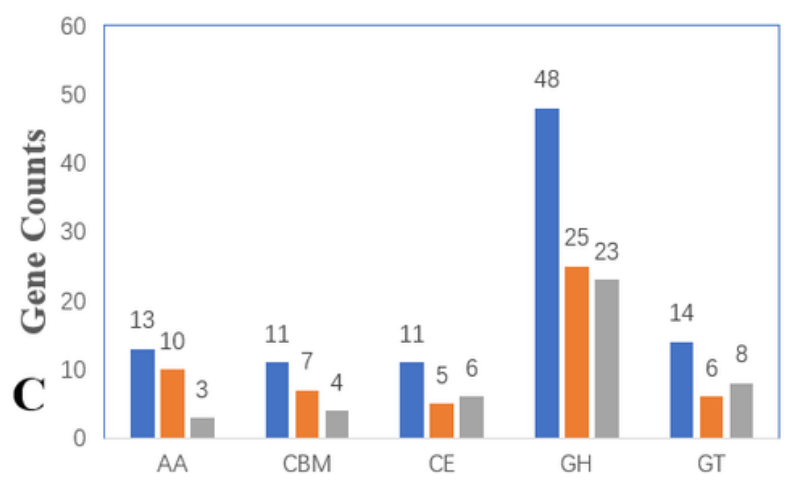

Fries3vsPD group

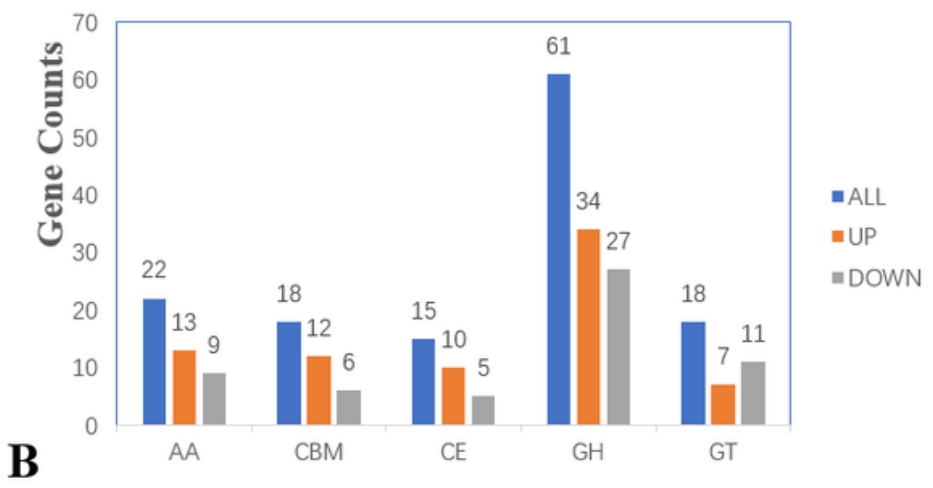

Fries3vsPS group

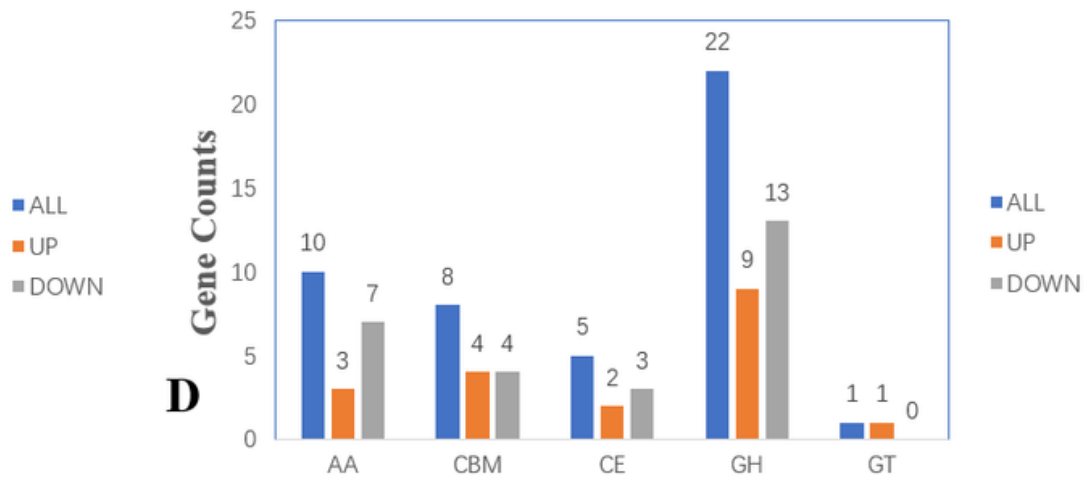

\section{Figure 7}

The CAZymes analysis and in the differential expression genes (A) The summary of the six CAZymes categories in the Trichoderma koningiopsis Transcriptome. (B) The CAZymes in the in the differential expression genes between Fries3 and PD. (C) (B) The CAZymes in the in the differential expression genes between PS and PD. (D) (B) The CAZymes in the in the differential expression genes between Fires3 and PD.

\section{Supplementary Files}

This is a list of supplementary files associated with this preprint. Click to download.

- suppepment20200807.docx 